demonstration * that all the roots of the polynomial solution are real, the reader is referred to an article by Bôcher in the April number of the Bullerrs. The method which he has there employed I shall make use of to prove that the roots of the accessory polynomial $\varphi$ are likewise real. Let $P$ denote the polynomial solution and $x_{1}, \cdots, x_{n-1}$ the roots of its derivative which are, of course, real. If $P$ be substituted in the differential equation and $x$ be placed equal to a root $\alpha$ of $\varphi$, we get

$$
P^{\prime \prime}(\alpha)+\left(\frac{1-\lambda_{1}}{\alpha-e_{1}}+\cdots+\frac{1-\lambda_{r}}{\alpha-e_{r}}\right) P^{\prime}(\alpha)=0,
$$

or dividing by $P^{\prime}(\alpha)$,

$$
\frac{1}{\alpha-x_{1}}+\cdots+\frac{1}{\alpha-x_{n-1}}+\frac{1-\lambda_{1}}{\alpha-e_{1}}+\cdots+\frac{1-\lambda_{r}}{\alpha-e_{r}}=0 .
$$

If now $\alpha$ is an imaginary root $p+q i$ for which $q$ is positive, the pure imaginary part of each fraction will have a negative sign. The equation therefore involves a contradiction. Hence

VIII. The roots of the accessory polynomial $\varphi$ of the differential equation (8) for a Stieltjes polynomial are all real and included between the two extreme singular points, $e_{1}$ and $e_{r}$.

WESLEYAN UNIVERSITY, April, 1898.

\title{
NOTE ON STOKES'S THEOREM IN CURVILINEAR CO-ORDINATES.
}

BY PROFESSOR ARTHUR GORDON WEBSTER.

(Read before the American Mathematical Society at the Meeting of April 30, 1898.)

THE expression for the curl of a vector point-function, when required in terms of orthogonal curvilinear coördinates, is usually obtained by direct transformation from their values in rectangular coördinates. The proof of Stokes's theorem given in my Lectures on electricity and magnetism, due to Helmholtz, can be easily adapted to curvilinear coördinates so as to prove the theorem independently of rectangular coördinates.

Let $P_{1}, P_{2}, P_{3}$ be the projections of a vector $P$ on the

* The proof given by Stieljes in the sixth volume of the Acta Mathematica is based upon mechanical considerations. 
varying directions of the tangents to the coördinate lines at any point. Let the projections in the same directions of the arc $d s$ of a curve connecting two points $A$ and $B$ be $d s_{1}$, $d s_{2}, d s_{3}$. The theorem concerns the line integral of the resolved tangential component of the vector along the given curve :

$$
\begin{gathered}
I=\int_{A}^{B} P \cos (P, d s) d s \\
=\int_{A}^{B}\left(P_{1} d s_{1}+P_{2} d s_{2}+P_{3} d s_{3}\right) .
\end{gathered}
$$

But in terms of the curvilinear coördinates $\rho_{1}, \rho_{2}, \rho_{3}$ we have

where

$$
d s_{1}=\frac{d \rho_{1}}{h_{1}}, \quad d s_{2}=\frac{d \rho_{2}}{h_{2}}, \quad d s_{3}=\frac{d \rho_{3}}{h_{3}},
$$

$$
h_{s}^{2}=\left(\frac{\partial \rho_{s}}{\partial x}\right)^{2}+\left(\frac{\partial \rho_{s}}{\partial y}\right)^{2}+\left(\frac{\partial \rho_{s}}{\partial z}\right)^{2} \quad(s=1,2,3) .
$$

Let us now make an infinitesimal transformation of the curve, so that the transformed curve shall lie on a given surface containing $A$ and $B$, and shall itself pass through those points. Then the change in the integral due to the infinitesimal changes $\delta \rho_{1}, \delta \rho_{2}, \delta \rho_{3}$ in the coördinates is

$$
\begin{gathered}
\delta I=\delta \int\left(\frac{P_{1}}{h_{1}} d \rho_{1}+\frac{P_{2}}{h_{2}} d \rho_{2}+\frac{P_{3}}{h_{3}} d \rho_{3}\right) \\
=\int\left[\delta\left(\frac{P_{1}}{h_{1}}\right) d \rho_{1}+\delta\left(\frac{P_{2}}{h_{2}}\right) d \rho_{2}+\delta\left(\frac{P_{3}}{h_{3}}\right) d \rho_{3}+\frac{P_{1}}{h_{1}} d \delta \rho_{1}\right. \\
\left.+\frac{P_{2}}{h_{2}} d \delta \rho_{2}+\frac{P_{3}}{h_{3}} d \delta \rho_{3}\right] .
\end{gathered}
$$

The last three terms can be integrated by parts, giving

$$
\int_{A}^{B} \frac{P_{s}}{h_{s}} d \delta \rho_{s}=\left.\frac{P_{s}}{h_{s}} \delta \rho_{s}\right|_{A} ^{B}-\int \delta \rho_{s} d\left(\frac{P_{s}}{h_{s}}\right) \quad(s=1,2,3),
$$

and, the integrated terms vanishing at the limits,

$$
\begin{gathered}
\delta I=\int\left[\delta\left(\frac{P_{1}}{h_{1}}\right) d \rho_{1}+\delta\left(\frac{P_{2}}{h_{2}}\right) d \rho_{2}+\delta\left(\frac{P_{3}}{h_{3}}\right) d \rho_{3}-d\left(\frac{P_{1}}{h_{1}}\right) \delta \rho_{1}\right. \\
\left.-d\left(\frac{P_{2}}{h_{2}}\right) \delta \rho_{2}-d\left(\frac{P_{3}}{h_{3}}\right) \delta \rho_{3}\right] .
\end{gathered}
$$


Performing the operations denoted by $\delta$ and $d$, six of the eighteen terms cancel, and there remain the terms

$$
\begin{aligned}
& \delta I=\int {\left[\left(\delta \rho_{2} d \rho_{3}-\delta \rho_{3} d \rho_{2}\right)\left\{\frac{\partial}{\partial \rho_{2}}\left(\frac{P_{3}}{h_{3}}\right)-\frac{\partial}{\partial \rho_{3}}\left(\frac{P_{2}}{h_{2}}\right)\right\}\right.} \\
&+\left(\delta \rho_{3} d \rho_{1}-\delta \rho_{1} d \rho_{3}\right)\left\{\frac{\partial}{\partial \rho_{3}}\left(\frac{P_{1}}{h_{1}}\right)-\frac{\partial}{\partial \rho_{1}}\left(\frac{P_{3}}{h_{3}}\right)\right\} \\
&\left.+\left(\delta \rho_{1} d \rho_{2}-\delta \rho_{2} d \rho_{1}\right)\left\{\frac{\partial}{\partial \rho_{1}}\left(\frac{P_{2}}{h_{2}}\right)-\frac{\partial}{\partial \rho_{2}}\left(\frac{P_{1}}{h_{1}}\right)\right\}\right]
\end{aligned}
$$

Now the changes $\delta \rho_{2}, d \rho_{2}, \delta \rho_{3}, d \rho_{3}$ in the coördinates correspond to distances

$$
\frac{\delta \rho_{2}}{h_{2}}, \quad \frac{d \rho_{2}}{h_{2}}, \quad \frac{\delta \rho_{3}}{h_{3}}, \quad \frac{d \rho_{3}}{h_{3}},
$$

measured along the coördinate lines, and the determinant of these distances

$$
\frac{1}{h_{2} h_{3}}\left\{\delta \rho_{2} d \rho_{3}-d \rho_{3} \delta \rho_{2}\right\}
$$

is equal to the area of the projection on the surface $\rho_{1}$ of the infinitesimal parallelogram swept over by the arc $d s$ during the transformation. Calling this area $d S$, and its normal $n$, we have

$$
\frac{1}{h_{2} h_{3}}\left(\delta \rho_{2} d \rho_{3}-\delta \rho_{1} d \rho_{3}\right)=\cos \left(n n_{1}\right) d S, \text { etc. }
$$

Now, repeating the transformation so that the given curve 1 is transformed into a second given curve 2 joining $A B$, the total change in the line integral is represented by the surface integral over the surface lying between the curves

$$
\begin{gathered}
\int \delta I=I_{2}-I_{1} \\
=\iint\left[h_{2} h_{3}\left\{\frac{\partial}{\partial \rho_{2}}\left(\frac{P_{3}}{h_{3}}\right)-\frac{\partial}{\partial \rho_{3}}\left(\frac{P_{2}}{h_{2}}\right)\right\} \cos \left(n n_{1}\right)\right. \\
+h_{3} h_{1}\left\{\frac{\partial}{\partial \rho_{3}}\left(\frac{P_{1}}{h_{1}}\right)-\frac{\partial}{\partial \rho_{1}}\left(\frac{P_{1}}{h_{3}}\right)\right\} \cos \left(n n_{2}\right) \\
\left.+h_{1} h_{2}\left\{\frac{\partial}{\partial \rho_{1}}\left(\frac{P_{2}}{h_{2}}\right)-\frac{\partial}{\partial \rho_{2}}\left(\frac{P_{1}}{h_{1}}\right)\right\} \cos \left(n n_{3}\right)\right] d S .
\end{gathered}
$$


But the difference of the line-integrals $I_{2}-I_{1}$ is the lineintegral around the closed contour 21, so that we have the line-integral of the tangential component of the vector $P$ around the closed contour proved equal to the surfaceintegral, over a surface bounded by the contour, of the normal component of a vector $\Omega$ whose components are

$$
\begin{aligned}
& \omega_{1}=h_{2} h_{3}\left\{\frac{\partial}{\partial \rho_{2}}\left(\frac{P_{3}}{h_{3}}\right)-\frac{\partial}{\partial \rho_{3}}\left(\frac{P_{2}}{h_{2}}\right)\right\}, \\
& \omega_{2}=h_{3} h_{1}\left\{\frac{\partial}{\partial h_{3}}\left(\frac{P_{1}}{h_{1}}\right)-\frac{\partial}{\partial \rho_{1}}\left(\frac{P_{3}}{h_{3}}\right)\right\}, \\
& \omega_{3}=h_{0} h_{2}\left\{\frac{\partial}{\partial \rho_{1}}\left(\frac{P_{2}}{h_{2}}\right)-\frac{\partial}{\partial \rho_{1}}\left(\frac{P_{1}}{h_{1}}\right)\right\} .
\end{aligned}
$$

The vector $\Omega$ is called the curl of $P$.

\section{ON THE STEINER POINTS OF PASCAL'S HEXAGON.}

BY DR. VIRGIL SYNDER.

The proof given by v. Staudt $*$ of the conjugate nature of $M, N$ with regard to the conic for which $M, N$ are associated Steiner points is perhaps rigorous, but unnecessarily long, and the most important statement $\dagger$ is only proved for the particular case in which the two triads of points defining the hexagon are linearly perspective.

He gives a second proof in article 8 of the same paper which is much shorter, but involves imaginary elements.

The following proof is much more simple and direct than either, and shows clearly which of Steiner's points are associated as "Gegenpunkte."

Let $A_{1}, A_{2}, A_{3}$ and $B_{1}, B_{2}, B_{3}$ be two triads of points lying on the same conic; these points can be made projective in six ways. namely

$$
\left(\begin{array}{l}
A_{1} A_{2} A_{3} \\
B_{1} B_{2} B_{3}
\end{array}\right) \quad\left(\begin{array}{l}
A_{2} A_{3} A_{1} \\
B_{1} B_{2} B_{3}
\end{array}\right) \quad\left(\begin{array}{l}
A_{3} A_{1} A_{2} \\
B_{1} B_{2} B_{3}
\end{array}\right)
$$

\footnotetext{
* Ueber die Steiner'schen Gegenpunkte ***, Crelle's Journal, vol. 62. $\dagger$ "Weil ferner $P, Q$ harmonïsch getrennt sind durch $M$ und seine Polare
} 